\title{
Design of Efficient Hybrid E/F Power Amplifier for Biomedical Purposes
}

\author{
Saad Mutashar \\ Department of Electrical Engineering, University of Technology, Iraq \\ Saad_ra25@yahoo.com,30099@uotechnology.edu.iq
}

\begin{abstract}
This paper introduces a new hybrid E/F power amplifier for subcutaneous applications. The proposed design is based on Amplitude Shift Keying (ASK) modulation technique with Industrial Scientific Medical (ISM) band operating at $135 \mathrm{kHz}$ to avoid the tissue damage. The Hybrid E/F power amplifier combines the tuning network circuits and uses a control unit to control a selectable switch to choose the desired amplifier. The Amplitude Shift Keying modulator is proposed to supply the proposed hybrid amplifier with approximately constant DC voltage. The proposed power amplifier aims to provide an appropriate power to power the implantable devices and reduce the hardware of the subcutaneous devices depending on the specific applications.
\end{abstract}

Index Terms - Class-E and F power amplifiers, Implantable Devices, Inductive Links, Control Units.

\section{INTRODUCTION}

The power amplifier plays a vital role in low and high-frequency bands. It amplifies the input signal to a particular power level, which drives the transmit network circuit. The power efficiency is one of the critical factors in the design of the Power Amplifier (PA). Ultimately, when it comes to the power efficiency class $\mathrm{E}$ and $\mathrm{F}$, amplifiers have a better output efficiency compared to these conventional amplifiers, such as class A, B, C, and D [1]. A concise comparison of the efficiency between different types of power amplifiers is shown in Table 1[2]. However, the principles of the power switch amplifiers that have been studied designed and which are appropriate for the biomedical purpose [2].

The power amplifier is a switching amplifier that converts a low-level analog input signal into a high power, and it consists of three main stages: the input switching stage, the power amplification stage, and the output filter stage [3], [4]. To achieve this technique a pulse width modulation (PWM) takes the input signal, and converts it into a higher (squire waveform) switching waveform, which uses a comparator. When the voltage at the inverting input is more significant than at the non-inverting input, the output voltage is low, and when the voltage at the inverting input smaller than that at the noninverting input, the output voltage becomes high [5]. The output wave of the power amplifiers suffered from weakness due to the misalignment or variation in distance between the transmitted and received coils, and to solve the above issue a feedback control technique is used [6,7]. Generally, there are three modulation techniques used in the biomedical applications such as Amplitude Shift Keying (ASK), Frequency Shift Keying (FSK) and Phase Shift Keying (PSK) where the ASK modulation technique is widely used in the implanted device due to its low power consumption and simple architecture design [8].

In general the class D, E and F power amplifiers are the most amplifiers used in biomedical applications due to their efficiency. The power amplifiers operated frequency must be with the ISM band to avoid the human tissue harm [8]. Many researchers and studies discussed and optimized the above amplifiers to increase the delivered power to the bio-implantable devices [9]-[14]. 
In this work, a proposed Hybrid E/F power amplifier combines the tuning network circuits and uses a selectable switch to choose the desired amplifier is presented. The operating frequency $135 \mathrm{KHz}$ is selected according to the ISM band to avoid the human tissue damage. The proposed ASK modulator is also introduced to supply the proposed amplifier with approximately constant DC voltage and provide a modulated signal to the implanted devices. The results show that the output waveform is a very stable sinusoidal wave and the delivered power is higher when the selectable switch is chosen to select the class-F amplifier.

TABLE 1. COMPARISON OF CLASS POWER AMPLIFIERS EFFICIENCY

\begin{tabular}{cc}
\hline Power Amplifiers Types & Approximate efficiency (Theoretical) \\
\hline Class A & $35 \%$ \\
Class B & $50 \%$ \\
Class C & $65 \%$ \\
Class D & $80 \%$ \\
Class E & $90-95 \%$ \\
Class F & $95 \%$ \\
\hline
\end{tabular}

\section{CLASS-E POWER AMPLIFIER THEORY AND DESIGN}

The first part of the Hybrid E/F Power Amplifier is the class-E power amplifier. In general, class $\mathrm{E}$ power amplifier technique is commonly used in wireless power transmission for biomedical and biotelemetry applications [2]. The implant system needs a highly efficient power amplifier to transmit the RF power signal, which modulates according to the control information from the reader, into the implant device. Hence, class $\mathrm{E}$ is a more suitable power amplifier as an element driver for the transmitter coil, with actual efficiency is about 90 95\% [3]. A simple class E consists of a single pole MOSFET transistor switch with a shunt capacitor $\mathrm{C}_{2}$ parallel with series circuit $\mathrm{R}, \mathrm{L}, \mathrm{C}_{1}$ that achieves a constant current from the supply source as shown in Fig. 1. The Pulse width modulation (PWM) or the pulse generator takes the input signal and converts it into a higher (square waveform) switching waveform, which uses as a comparator as shown in Fig. 2.

For optimum class-E PA design, a straightforward analysis approach is produced. This analysis aims to determine the shunt capacitor, which plays an essential role in class-E design. In Figure 2, assuming the MOSFET transistor (S) is turned $\mathrm{ON}$ with a $50 \%$ duty cycle. The switch control signal Vg imposes the amplifier swapping between $\mathrm{ON}$ and $\mathrm{OFF}$ states. When the switch in ON state, the current is flowing across the switch and shorts the shunt capacitor $\mathrm{C} 2$; when the switch in OFF state. In order to avoid the power dissipation due to either shorting the shunt capacitor, or voltage drop, which is crossed it, during the switching or when the MOSFET turns ON.

When the class-E amplifier reaches the steady state hence, the averaged voltage across the switch is equal the inductor chock voltage $\left(\mathrm{V}_{\mathrm{ch}}\right)$. This makes the chosen value of the shunt capacitor is sensitive to the tank load and duty cycle. The larger tank load and larger turn off duty cycle need smaller $\mathrm{C}_{2}$ and more sensitive to the parasitic capacitance. The component values of class-E are shown in Table 2 and calculated as follow. As a first order estimation, when the duty cycle is $50 \%$, the shunt capacitor calculated as given in (1) and the series capacitor $\mathrm{C}_{1}$ expressed as given in (2) [3].

$$
C_{2}=\frac{1}{\omega\left(5.447 R_{\text {load }}\right)}
$$

Where $\quad \omega=2 \pi f$ 


$$
C_{1}=C_{2}\left[\frac{5.447}{Q}\right]\left[1+\frac{1.42}{Q-2.08}\right]
$$

The values of $\mathrm{L}_{\mathrm{ch}}$ can be found as given in (3) [8].

$$
L_{\text {chock }}=\frac{7 R_{\text {load.opt }}}{f}
$$

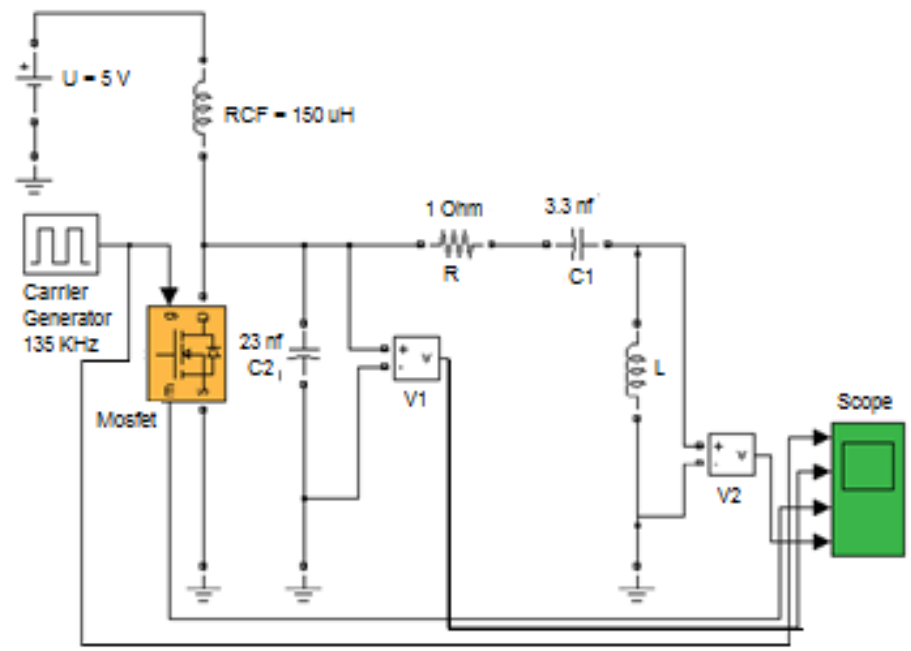

FIG.1. THE SIMULATION OF CLASS-E-PA WITH MATLAB/SIMULINK

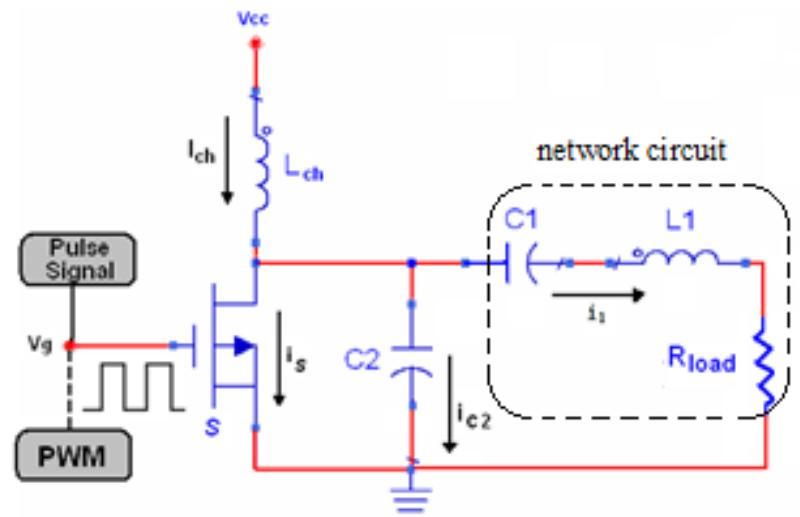

FIG. 2. THE CLASS-E AMPLIFIER WITH NETWORK

To obtain high power output drain efficiency, the quality factor of the self-inductance (coil $\mathrm{L}_{1}$ ) should be high and chosen $\mathrm{Q}=100$, and the value of $\mathrm{k}$ is equal 1.4 [15]. However, the quality factor of the load network affects the bandwidth of the RF transmitting signal to the reader and calculated as follows:

$$
Q_{\text {coil }}=\frac{\omega L_{\text {match }}}{R_{\text {coil }}}
$$

The quality factor of the tuned circuit (Load Network) in Fig.1 can be obtained by the equation below as;

$$
\begin{gathered}
Q_{\text {network }}=\frac{\sqrt{\frac{L_{1}}{C_{2}+C_{1}}}}{R_{\text {load }}} \\
L_{1}=\frac{Q R_{L}}{\omega}
\end{gathered}
$$


TABLE 2. THE COMPONENT VALUES OF CLASS-E PA

\begin{tabular}{ccccc}
\hline Choke. $(\boldsymbol{\mu} \mathbf{H})$ & $\mathbf{C}_{\mathbf{2}}(\mathbf{n f})$ & $\mathbf{R}(\mathbf{\Omega})$ & $\mathbf{C}_{\mathbf{1}}(\mathbf{n f})$ & Transmit Coil $(\boldsymbol{\mu} \mathbf{H})$ \\
\hline 150 & 23 & 1 & 3.3 & 150 \\
\hline
\end{tabular}

\section{CLASS-F POWER AMPLIFIER AND DESIGN}

The second part of the Hybrid E/F Power Amplifier is the class-F power amplifier. Figure 3 shows the class-FPA which is commonly used to amplify the RF signals, which is given a high efficiency and low power consumption and dissipation: it can achieve a higher power compared to other traditional class amplifiers [16]. The fundamental operation of a class-F amplifier is similar to Class E PA as a switch power amplifier. The input square wave signal drain voltage is rich in odd harmonics the tuned filter network will remove the odd harmonics and the output RF signal, that provides a stable sine wave. Generally, it consists of three parts, the input matching circuit, switch transistor, and a filter network circuit. The output filter is used to control the harmonic content of its drain voltage or drain current waveforms thereby shaping them to reduce power dissipation by the transistor, thus allowing removal with a simple filter. The passive load network is designed to minimize current and voltage overlapping, and for overall efficiency, the switch frequency is much higher than the input signal band, which allows high frequency out of band components to be removed with a simple filter. The component values of class-F PA is given in Table 3, where the $L_{1}$ and $C_{2}$ tuned at a central frequency where the tuned circuit at the fundamental resonance can be defined in the mathematical expression as;

$$
\begin{gathered}
L_{1}=\frac{R}{\omega Q} \\
C_{1}=\frac{Q}{\omega R}
\end{gathered}
$$

The accurate removal harmonics from the input square wave signal gives the accurate sine wave output form with the LC tank circuit components. The transmitter coil voltage and the current in Fig. 3 can be defined as;

$$
\begin{gathered}
V_{\text {coil }}=\frac{2 V_{C C}}{\pi} \sin \omega t \\
I_{\text {coil }}=\frac{2 V_{C C}}{\pi \cdot R} \cos \omega t
\end{gathered}
$$




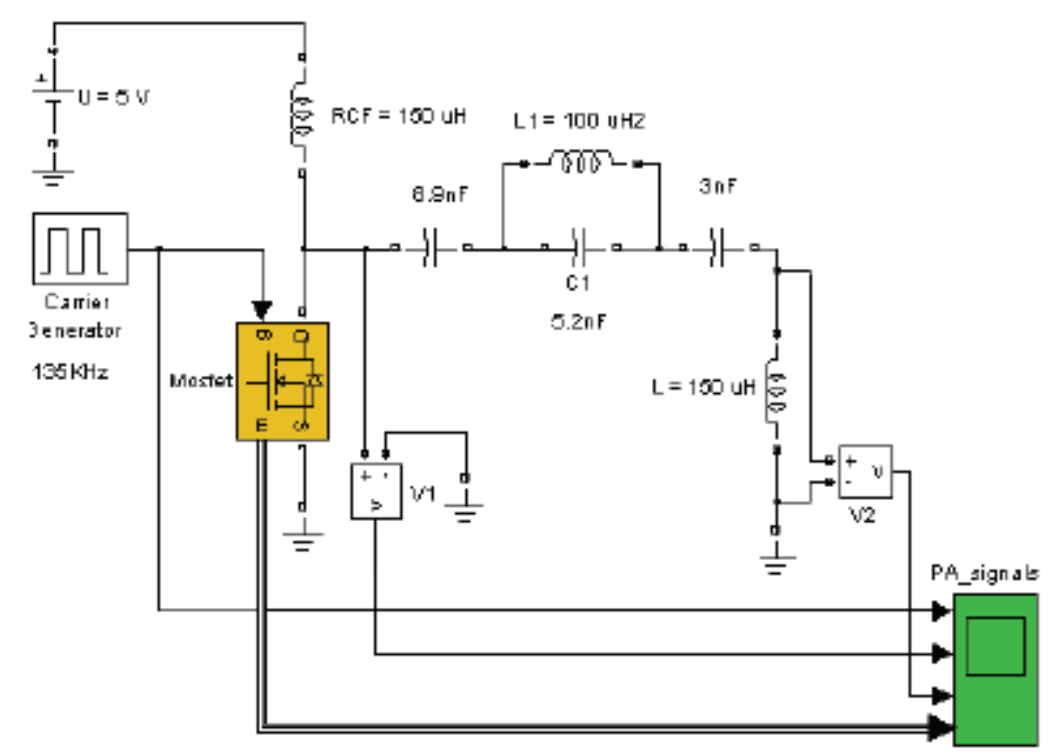

FIG.3. THE SIMULATION OF CLASS-F-PA WITH MATLAB/SIMULINK

TABLE 3. THE COMPONENT VALUES OF CLASS-F PA

\begin{tabular}{cccccc}
\hline Chock. $(\boldsymbol{\mu} \mathbf{H})$ & $\mathbf{C}(\mathbf{n f})$ & $\mathbf{C}_{\mathbf{1}}(\mathbf{n f})$ & $\mathbf{C}_{\mathbf{2}}(\mathbf{n f})$ & $\mathbf{L}_{\mathbf{1}}(\boldsymbol{\mu} \mathbf{H})$ & Transmit Coil $(\boldsymbol{\mu H})$ \\
\hline 150 & 6.9 & 5.2 & 3 & 100 & 150 \\
\hline
\end{tabular}

\section{PROPOSED HYBRID E/F POWER AMPLIFIER DESIGN}

In this study, a new hybrid power amplifier (HPA) designed by OrCAD PSpice 16.2 software that uses the control technique to improve the power efficiency of the power amplifier is introduced as shown in Fig. 4. The control unit technique is used to convert the output wave signal from the sine wave into a square wave. This control unit can be a microcontroller PWM generator or conventional methodology using PLL-VCO which can be controlled by a control logic circuit (CLC), which is capable of controlling the selectable amplifier $\mathrm{E}$ or $\mathrm{F}$. The controller adjusts the supply voltage of the hybrid $\mathrm{E} / \mathrm{F}$ power amplifier. The duty cycle (D) of the PWM pulse is controlled by the PWM generator which is proportional to the RF input signal.

The proposed Hybrid-PA combines the tuning network circuits and uses a selectable switch to choose the desired amplifier. In this work, the proposed ASK modulator is synthesized for driving the hybrid power amplifier by approximately constant DC voltage and additionally provides wireless power, whether data is present or not. The proposed Architecture of the ASK modulator is simple and involves two MOSFETs $\left(\mathrm{M}_{1}\right.$ and $\left.\mathrm{M}_{2}\right)$ and can receive pseudo-random binary numbers (PN) or data from a personal computer (PC).

Typically, the CLC drives the relay circuit. Furthermore, the feedback loop circuit captures the transmitted RF signal modulated or un-modulated, to monitor the carrier frequency signal, and perform the changeover between both amplifiers. The truth table demonstrates the state logic to control the desired amplifier can be explained in Table 4 . The suggested criteria which make the controller switch between the class $\mathrm{E}$ and $\mathrm{F}$ power amplifier is can be performed by implementing the logic control circuit with VHDL code to perform the function to control switching. 


\section{TABLE 4. THE TRUTH TABLE OF THE PROPOSED CONTROL UNIT}

\begin{tabular}{ccccc}
\hline Enc-E/F & Relay 1 & Relay 2 & Relay 3 & Relay 4 \\
\hline E-on & 1 & 1 & 1 & PN/PC \\
E-off & 0 & 0 & 0 & PN/PC \\
F-on & 1 & 1 & 1 & PN/PC \\
F-off & 0 & 0 & 0 & PN/PC \\
\hline
\end{tabular}

Eventually, the control command data can be interfaced from the PC to the reader through the ASK modulator, and also it can select one of the power amplifiers class $\mathrm{E}$ or $\mathrm{F}$. However, the main goal of synthesis the hybrid power amplifier is to provide a suitable power delivered to the implantable devices and reduce the hardware circuit.

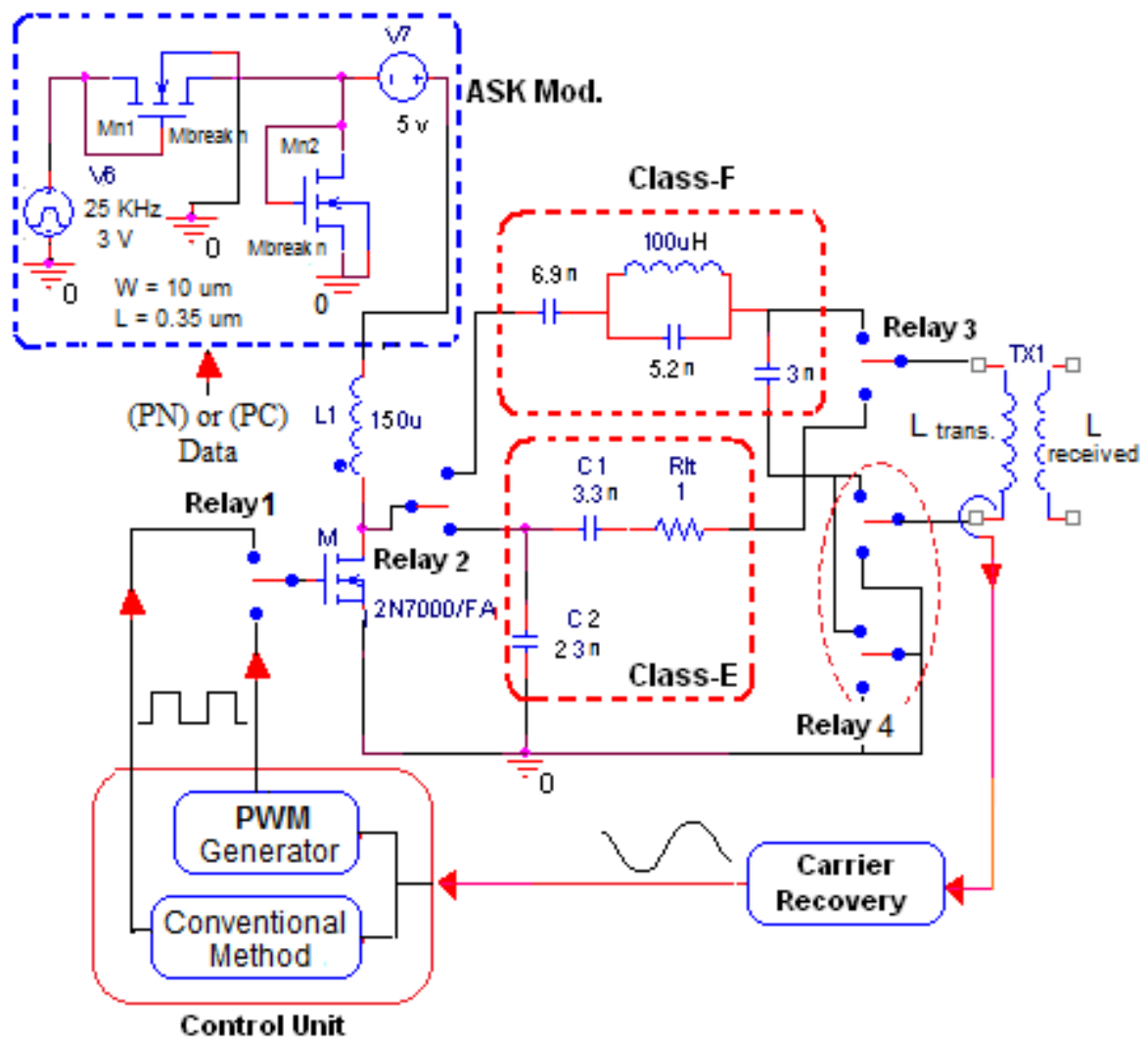

FIG. 4. THE PROPOSED HYBRID E/F PA WITH ASK MODULATOR

\section{RESULTS AND DISSECTIONS}

This paper deals to design a proposed hybrid $\mathrm{E} / \mathrm{F}$ power amplifier for subcutaneous applications based on ASK modulation technique with operating frequency $135 \mathrm{KHz}$. The main goal was to synthesize a highly efficient amplifier, which is reliable for medical purposes. Eventually, many parameters have to be considered in the design such as frequency stability and maximum transfer power. The switching power amplifier is chosen in this work, principally, the class $\mathrm{E}$ and Class $\mathrm{F}$ power amplifiers were studied and investigated in detail and simulated them with MATLAB/Simulink tools. However, the above two-switch power amplifiers were selected by combining these two power amplifiers in a hybrid design, to improve the performance efficiency of the transmitter power amplifier. 
The output wave of the proposed hybrid power amplifiers suffered from weakness due to the misalignment or variation in distance between the transmitted and received coils, and to solve the above issue a feedback control technique is used to convert the output wave signal from the sine wave into a triangular wave. This control unit can be a microcontroller PWM generator or conventional methodology to control and select the desired amplifier. Besides, the proposed ASK modulator is synthesized for driving the hybrid power amplifier with approximately stable DC voltage $\approx 5 \pm 1$ Vas shown in Fig. 5.

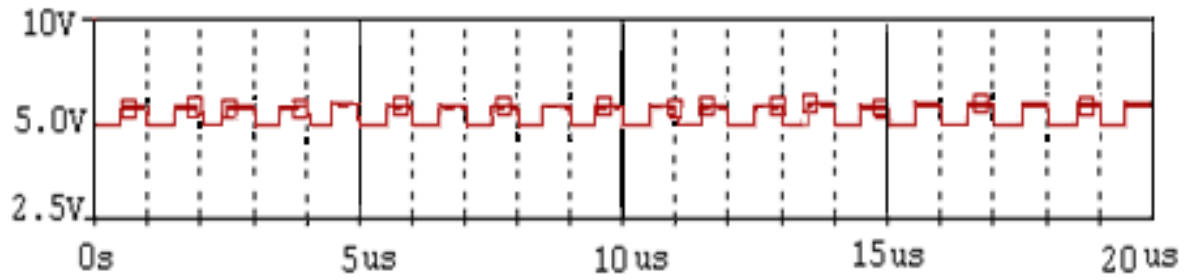

FIG. 5. THE DELIVERED DC VOLTAGE FROM ASK MODULATOR TO THE HYBRID E/F PA USING ORCAD PSPICE SOFTWARE

The MATLAB/Simulink environment is used to simulate and evaluate the performance of the class-E power amplifier module. Figure 6 shows the stable sinusoidal output waveform the carrier frequency is $135 \mathrm{KHz}$ with amplitude $\left(340 \mathrm{~V}_{\mathrm{pp}}\right)$ or $\left(\mathrm{V}_{\mathrm{rms}} 120 \mathrm{~V}\right)$ at the drain supply voltage is approximately constant at $5 \mathrm{~V}$. The transmit power, in this case, is 2 Watt.
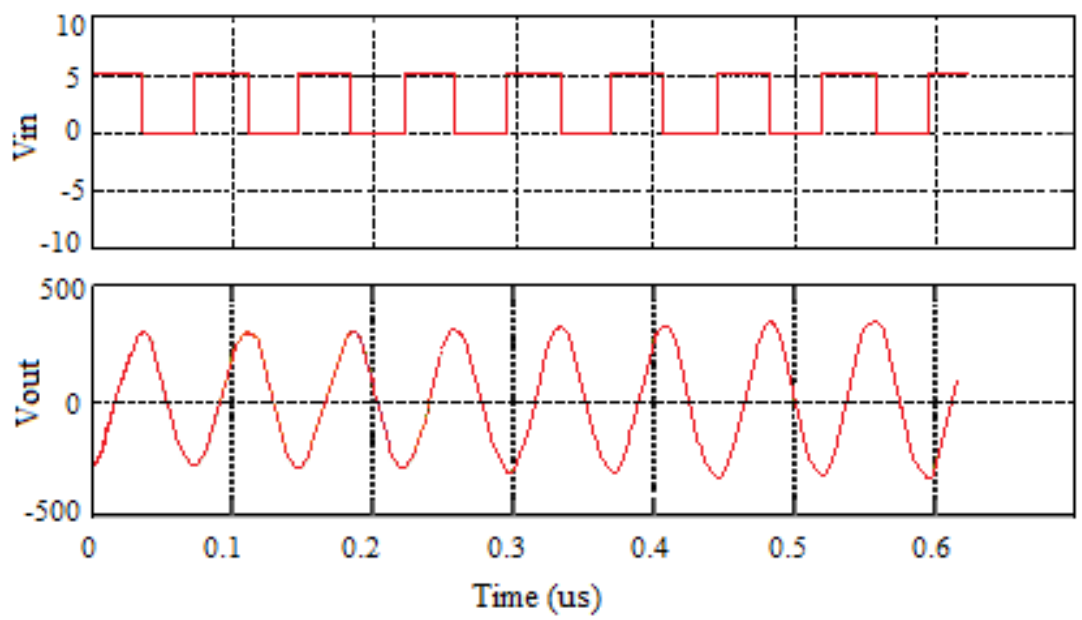

FIG. 6. THE SIMULATION RESULT WAVEFORM OF CLASS-E PA AT FREQUENCY 135 KHZ

The module of the class $\mathrm{F}$ power amplifier was also simulated and evaluated by using the same tools MATLAB/Simulink environment. Figure 7 shows the stable sinusoidal output waveform with $135 \mathrm{KHz}$ of the carrier frequency with amplitude $\left(397 \mathrm{~V}_{\mathrm{pp}}\right)$ or $\left(\mathrm{V}_{\mathrm{rms}}\right.$ $140 \mathrm{~V}$ ) at the drain supply voltage is approximately constant at $5 \mathrm{~V}$. The transmit power, in this case, is 3 Watt.

To improve the performance efficiency of the transmitter power amplifier and reduce the hardware layout. The above class-E and class-F power amplifiers were merged in one Hybrid E/F power amplifier combines the tuning network circuits and four relays and uses a control unit to control a selectable switch to choose the desired amplifier. 


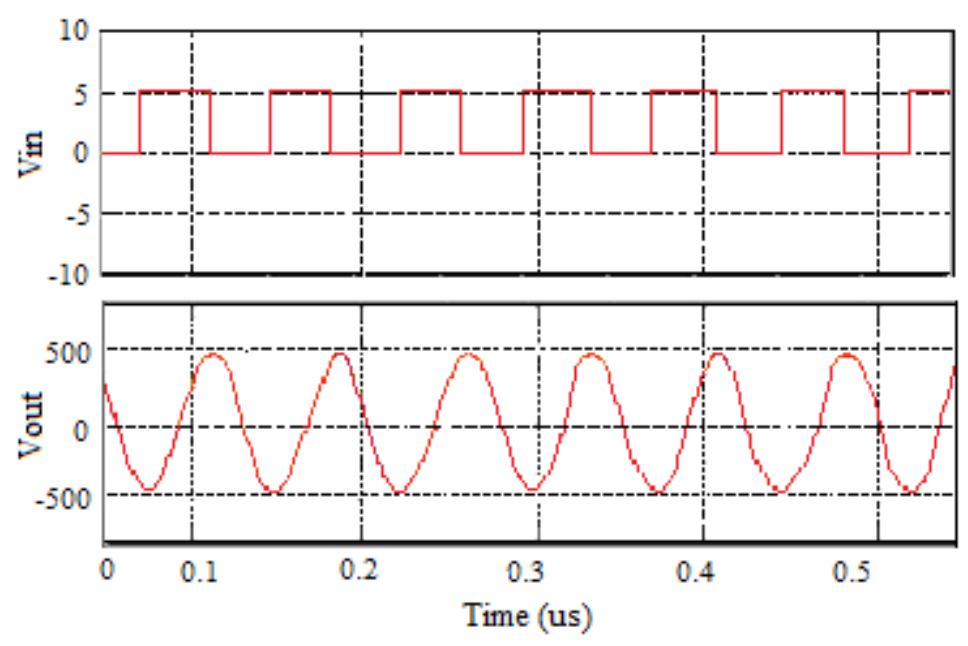

FIG. 7. THE SIMULATION RESULT WAVEFORM OF CLASS-F PA AT FREQUENCY 135 KHz

The feedback control system was synthesized with microcontroller PWM techniques or conventional methodology and additional circuits were designed to convert the transmitted RF signal into a digital RF signal. Because of the coils misalignment, alteration in distances TX between the transmitting coil $\left(\mathrm{L}_{\text {transmiter }}\right)$ and received coil $\left(\mathrm{L}_{\text {receiver }}\right)$ has appeared. Hence the frequency was changed with respect to the distance where the carrier frequency shifted from $135 \mathrm{kHz}$ up to $142 \mathrm{kHz}$, especially when the coils were moved closer together (1$2 \mathrm{~cm})$. Figure 8 shows the relationship between the delivered power to the implanted coil (receiver) and distances. From the plotting simulation, the comparing of the received power between Class $\mathrm{E}$ and $\mathrm{F}$ power amplifiers in the Hybrid $\mathrm{E} / \mathrm{F}$ shows that the efficiency when Class-F used is better than class-E. The proposed system is suitable for implantable nerves stimulator, cochlear implant and retinal implants where the distances between the coils less than $2 \mathrm{~cm}$.

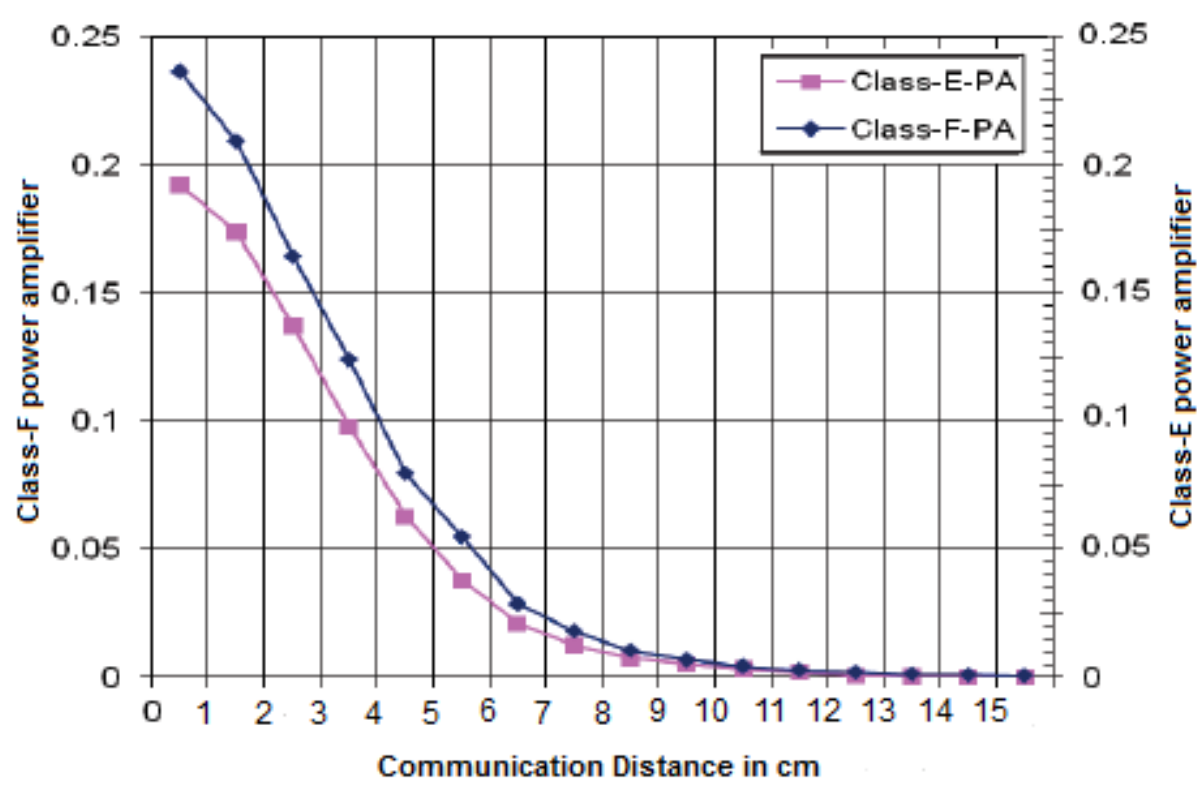

FIG. 8. THE COMPARISON OF THE RECEIVED POWER BETWEEN CLASS E AND F POWER AMPLIFIER 


\section{CONCLUSION}

This study presents a new design of hybrid E/F power amplifier for biomedical purposes. The proposed design based on ASK modulation technique with Industrial Scientific Medical (ISM) band at operating frequency $135 \mathrm{KHz}$ to avoid the tissue damage. The design consists of class-E and class-F power amplifiers and involves the tuning network circuits. The control unit is used to control and select the desired switch to choose the desired amplifier. The aim of the proposed power amplifier is to provide a suitable power to the implantable devices by using mutable power amplifiers. The simulation results show that the sinusoidal output waveform is very stable and the transmitted power is enough to power the implantable devices where class$\mathrm{F}$ has better efficiency than class-E. The proposed system is suitable for implantable nerves stimulator, cochlear implant, and retinal implants.

\section{REFERENCES}

[1] H. XIN. "Fully Integrated Transceiver Design in Soi Process, "Ph.D. thesis USA.Kansas State University 2004.

[2] S.M. Abbas, M.A. Hannan, and A.S. Salina, "Efficient class-E design for inductive powering wireless biotelemetry," applications, IEEE Int Conf of Biomedical Engineering (ICoBE), Malaysia, pp. 454_449, Perlis, 2012.

[3] N. O. Sokal, A. D. Sokal, "Class-E a new class of high efficiency tuned single-ended Switching power amplifiers," IEEE Journal of Solid-state circuit, vol. 10, pp.168-176, Jun 1975.

[4] F. H. Raab, "Effects of circuit variations on class E tuned power amplifier," IEEE J. of Solid-State Circuits, vol. 13, pp. 239-247. 1978.

[5] J.P. Carmo, P.M. Mendes. C. Couto, and J.H. Correia, "Low-power low-voltage RF COMS transceiver at2.4GHz" 5th telecommunication conference," Portugal, 5-6.April.2005.

[6] S. Mutashar, M.A. Hannan, A.S. Salina, and A. Hussain."Inductive coupling links for lowest misalignment effects in transcutaneous implanted devices". J Biomed Eng vol. 59, pp. 257-268. 2014.

[7] A. H. Hussein, M A Hannan, S. Mutashar, S. A Samad and A. Hussain. "Automatic Frequency Controller for Power Amplifiers Used in Bio-Implanted Applications: Issues and Challenges," Sensors. vol 14, pp. 23843-23870. 2014.

[8] S. Mutashar, M. A. Hannan, S. A. Samad, and A. Hussain, "Efficient Data and Power Transfer for Bio-Implanted Devices Based on ASK Modulation Techniques," Journal of Mechanics in Medicine and Biology (JMMB). Vol. 11, No 5, pp. 1240030. 2012

[9] A. N. Laskovski, M. R. Yuce, “Analysis of Class-E Amplifier with mixed data Modulation for Biotelemetry,” IEEE 29th international Conference of engineering in medicine and biology society, Lyon. France 22-26 Aug. 2007.

[10] M. K. Kazimierczuk, "Class E tuned power amplifier with the nonsinusoidal output voltage," IEEE J. of Solid-State Circuits, vol. 21, pp. 575-581, August 1986.

[11] S. Mutashar, M. A. Hannan, S. A. Samad, and A. Hussain, "Efficient Low-Power Recovery Circuits for Bio-implanted Micro-Sensors," Przeglad elektrotechniczny (ERJ), 89(5): 15-18. 2013.

[12] A. N. Laskovski, M. R Yuce, “Class-E Oscillators as wireless power Transmitters for Biomedical implants,” IEEE 3rd international technologies. Symposium on applied sciences in biomedical and communication Roma-Italy, 2010.

[13] N. Furqan, D. Maeve, “Amplifier design for a biomedical inductive power system," IEEE 21st international conference on signals and systems. Cork Ireland, pp. 169-174, Jun 2010.

[14] M. Alghrairi1, N. B. Sulaiman1, R. B. M. Sidek1, and S. Mutashar, "Unique Micro System Stimulator with High Data Rate and Efficient Power Recovery Circuit," Przeglad elektrotechniczny (ERJ) 92 (11). 2016.

[15] S. Mutashar, M A Hannan, S. A Samad and A. Hussain, "Analysis of Transcutaneous Inductive Powering Links," IEEE 4th International Conference on Intelligent and Advanced Systems (ICIAS) Kuala Lumpur, Malaysia.12-14, pp. 553-556. June 2012;

[16] S. Theodore,Rappaport, "Wireless Communications Principles and Practices" 2nd edition Pp 294 304. 2012. 